\title{
Multidimensional optical fractionation with holographic verification
}

\author{
Ke Xiao and David G. Grier \\ Department of Physics and Center for Soft Matter Research, New York University, New York, NY 10003
}

(Dated: October 19, 2018)

\begin{abstract}
The trajectories of colloidal particles driven through a periodic potential energy landscape can become kinetically locked in to directions dictated by the landscape's symmetries. When the landscape is realized with forces exerted by a structured light field, the path a given particle follows has been predicted to depend exquisitely sensitively on such properties as the particle's size and refractive index These predictions, however, have not been tested experimentally. Here, we describe measurements of colloidal silica spheres' transport through arrays of holographic optical traps that use holographic video microscopy to track individual spheres' motions in three dimensions and simultaneously to measure each sphere's radius and refractive index with part-per-thousand resolution. These measurements confirm previously untested predictions for the threshold of kinetically locked-in transport, and demonstrate the ability of optical fractionation to sort colloidal spheres with part-per-thousand resolution on multiple characteristics simultaneously.
\end{abstract}

Optical fractionation is akin to sifting particulate matter with a sieve made of light [1, 2, 3]. Colloidal particles flowing with or through a viscous medium such as water are deflected into different directions by their differing interactions with a structured light field 1]. Unlike photophoresis [4], in which particles are deflected down the optical axis by radiation pressure, optical fractionation relies on optical forces directed transverse to the optical axis [5, [6] and on the symmetries and periodicities of the structured light field to select different fractions from an

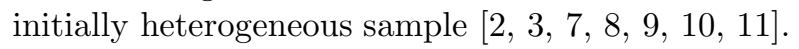

Pioneering studies [1, 2, 3, 7, 9, 11] have suggested that optical fractionation may offer unparalleled selectivity for particle size and refractive index. Lacking sufficiently precise methods to test these predictions directly, experimental studies either have focused on sorting polydisperse samples of particles with easily distinguishable properties [2, 3, 12] or else have used indirect approaches to assess the method's finesse [13].

This Letter describes optical fractionation measurements on nominally monodisperse colloidal samples whose performance is verified directly using the highresolution single-particle characterization and tracking capabilities 14] of holographic video microscopy 15]. These measurements demonstrate optical fractionation on the basis of size and refractive index with part-perthousand resolution and agree quantitatively with predictions derived from limiting arguments for colloidal transport through optical trap arrays [3, 7]. They thus cast new light on the robustness of kinetically locked-in transport against the inevitable disorder in practical implementations.

Our experimental system is depicted schematically in Fig. 1. Aqueous dispersions of colloidal silica spheres are driven by a syringe pump (Harvard Apparatus PHD 2000) at a constant flow rate of $4 \mu \mathrm{l} / \mathrm{h}$ through a rectangular microfluidic channel formed by bonding a poly(dimethylsiloxane) casting to a glass microscope cover slip [16]. The resulting channel is $3 \mathrm{~cm}, 30 \mu \mathrm{m}$

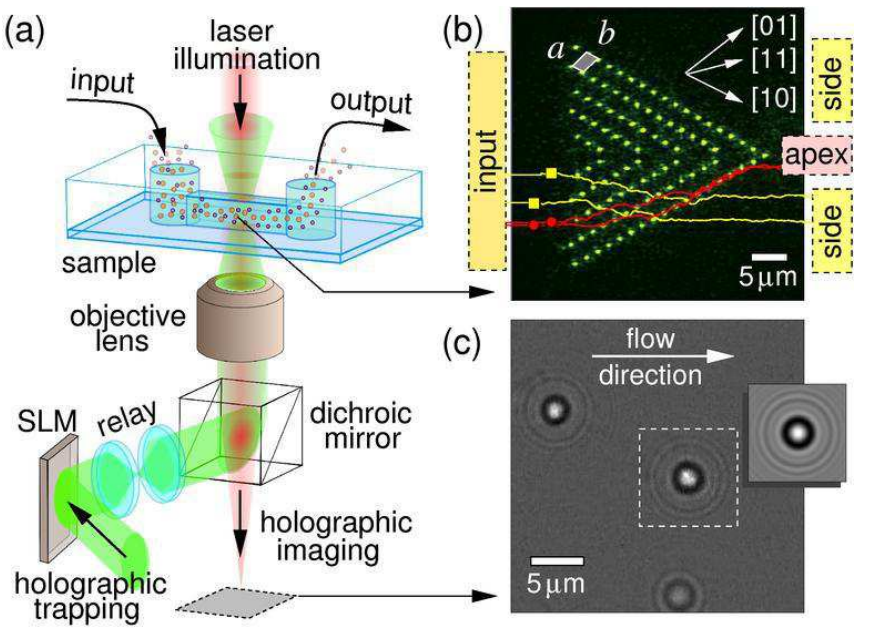

FIG. 1: (color online)(a) Combined holographic optical trapping and holographic video microscopy system for tracking and characterizing colloidal spheres during optical fractionation. The objective lens focuses computer-generated holograms projected with a spatial light modulator (SLM) into the sample. It also collects holographic images of the sample that are recorded with a video camera. Trapping and imaging light are separated with a dichroic mirror. (b) Image of 103 holographic optical tweezers in the microscope's focal plane. The traps are arranged in two domains of oblique lattice points with $a=3.27 \mu \mathrm{m}$ and $b=2.025 \mu \mathrm{m}$. One unit cell is highlighted. The domains are oppositely inclined so that $\theta_{[10]}= \pm 30^{\circ}, \theta_{[01]}=\mp 38^{\circ}$ and $\theta_{[11]}=\mp 13^{\circ}$ with respect to the flow direction. Dashed boxes indicate input and output regions. Traces show trajectories for four representative particles measured at $1 / 30 \mathrm{~s}$ intervals. (c) Normalized holographic image of $1.5 \mu \mathrm{m}$ diameter silica spheres in the same field of view. Inset: Fit to the Lorenz-Mie theory of the outlined sphere's holographic image.

deep and $1 \mathrm{~mm}$ wide. Spheres travel along the channel in a parabolic Poiseuille flow profile, their speed depending on their height in the channel.

The flowing spheres interact with an array of holographic optical traps [17, 18] whose focused intensity 
pattern is recorded in Fig. 1(b). The pattern of 103 optical tweezers [5] is created from a single laser beam by imprinting a computer-generated hologram [18] onto its wavefronts using a phase-only spatial light modulator (SLM; Hamamatsu X8267-16). The hologram is relayed to the objective lens (Nikon Plan-Apo, 100×, NA 1.4, oil immersion) of an inverted optical microscope (Nikon TE2000U), which focuses it into diffraction-limited traps at the midplane of the sample [19]. Figure1(b) was created by placing a front-surface mirror in the microscope's focal plane, collecting the reflected light with the objective lens, and recording it with the microscope's video camera (NEC TI-324A II). The traps are powered by a solid state laser (Coherent Verdi $5 \mathrm{~W}$ ) operating at a vacuum wavelength of $\lambda=532 \mathrm{~nm}$.

The optical tweezer array was designed to sort an input distribution of colloidal spheres into two fractions that are kinetically locked in to two distinct lattice directions, and thus are deflected by the array in opposite directions. The array consists of two oblique lattice domains whose [10] axes are oppositely inclined at $\theta_{[10]}= \pm 30^{\circ}$ with respect to the flow direction. Photometry performed on the traps' images suggests that their intensities vary by roughly $10 \%$. This is consistent with calibration measurements based on the thermally-driven fluctuations of spheres trapped in each tweezer under zero-flow conditions [18, 20]. Trajectories that become kinetically locked in to the [10] direction, such as the two measured examples denoted by circles in Fig. 1(b), are deflected toward the apex of the array before flowing downstream. Other particles that interact too weakly with the traps to be deflected by $30^{\circ}$ may still become locked in to the [11] direction, which is inclined at $\theta_{[11]}=\mp 13^{\circ}$ in the two domains. Particles traveling along this direction are deflected away from the apex to the sides of the array, as indicated by the two typical trajectories denoted by squares in Fig. 1(b). The static array of traps therefore sorts the sample into two distinct fractions, one concentrated at the apex of the array, and the other deflected to its sides.

The objective lens also is used to holographically track and characterize individual spheres as they travel through the array. To do this, the sample volume is illuminated with the collimated $3 \mathrm{~mm}$ diameter beam from a HeNe laser operating at a vacuum wavelength of $632.8 \mathrm{~nm}$. The laser's irradiance is on the order of $1 \mathrm{nW} / \mu \mathrm{m}^{2}$, which is less than $10^{-4}$ of an individual trap's irradiance and is comparable to that of a conventional incandescent illuminator. Light scattered by a sphere interferes with the unscattered portion of the beam to form an interference pattern in the microscope's focal plane. This is magnified and its intensity recorded by the video camera with a calibrated spatial resolution of $91 \mathrm{~nm} /$ pixel at 30 frames/s. Figure 1(c) shows a detail of a typical holographic snapshot of $1.5 \mu \mathrm{m}$ diameter silica spheres normalized by a background image to eliminate speckle and other static nonuniformities [14].
Each snapshot is analyzed [14] using the Lorenz-Mie theory of light scattering [21] to obtain each colloidal sphere's three-dimensional position, $\boldsymbol{r}_{p}(t)$, relative to the center of the focal plane at time $t$, its radius $a_{p}$, and its complex index of refraction, $n_{p}$. Rigorous error estimates 14] yield nanometer resolution for $\boldsymbol{r}_{p}$ and $a_{p}$, and part-per-thousand resolution for $n_{p}$. Such precise singleparticle characterization makes possible a direct test of optical fractionation's resolution.

A particle becomes kinetically locked-in to one of the array's crystallographic directions because its interaction with one trap deflects it toward the next trap along that direction, and so on through the lattice [1, 3, 7, 9, 11, 22]. Modeling an individual trap as a Gaussian potential well of width $\sigma_{p}$ and depth $V_{p}$ separated from its neighbors by a distance $b$, the maximum angle $\theta_{\max }$ by which a particle driven by force $\boldsymbol{F}_{p}$ can be deflected has been estimated [3, 7, 13] to satisfy

$$
\theta_{\max } \leq \sin ^{-1}\left(\frac{2}{\sqrt{e}} \frac{V_{p}}{\sigma_{p} F_{p}} \exp \left(-\frac{b^{2}}{8 \sigma_{p}^{2}}\right)\right) .
$$

This is a general result that applies equally well to arrays of potential barriers as to potential wells [7, 22]. In the particular case of a micrometer-scale colloidal sphere interacting with optical tweezers, the effective well depth depends on both the particle's size and refractive index, and is given approximately by [7, 23],

$$
V_{p}=\frac{n_{m} a_{p}^{3}}{\sigma_{p}^{2} c}\left(\frac{n_{p}^{2}-n_{m}^{2}}{n_{p}^{2}+2 n_{m}^{2}}\right) P,
$$

where $n_{m}$ is the refractive index of the medium and $P$ is the laser power in an individual trap. The well's effective width [3, 7], $\sigma_{p}^{2}=a_{p}^{2}+\lambda^{2} /\left(4 n_{m}^{2}\right)$, also depends on the particle's size because larger spheres encounter a trap's localized light field from greater distances. Finally, the driving force due to Stokes drag in a fluid of viscosity $\eta$ flowing at velocity $\boldsymbol{v}$ is

$$
\boldsymbol{F}_{p}=6 \pi \eta a_{p} \boldsymbol{v}
$$

assuming that hydrodynamic coupling to boundaries and other spheres is weak enough to ignore [24].

Equations (11), (2) and (3) define conditions for marginally locked-in transport that divide the $\left(a_{p}, n_{p}\right)$ plane into a class of particles that can be deflected to angle $\theta$ and a complementary class that cannot. This division falls along the curve

$$
\begin{aligned}
& n_{c}\left(a_{p}\right)=n_{m} \sqrt{\frac{A\left(a_{p}\right)+2 B}{A\left(a_{p}\right)-B}}, \text { where } \\
& A\left(a_{p}\right)=\left(\frac{a_{p}}{b}\right)^{2}\left(\frac{b}{\sigma_{p}}\right)^{3} \exp \left(-\frac{b^{2}}{8 \sigma_{p}^{2}}\right)
\end{aligned}
$$



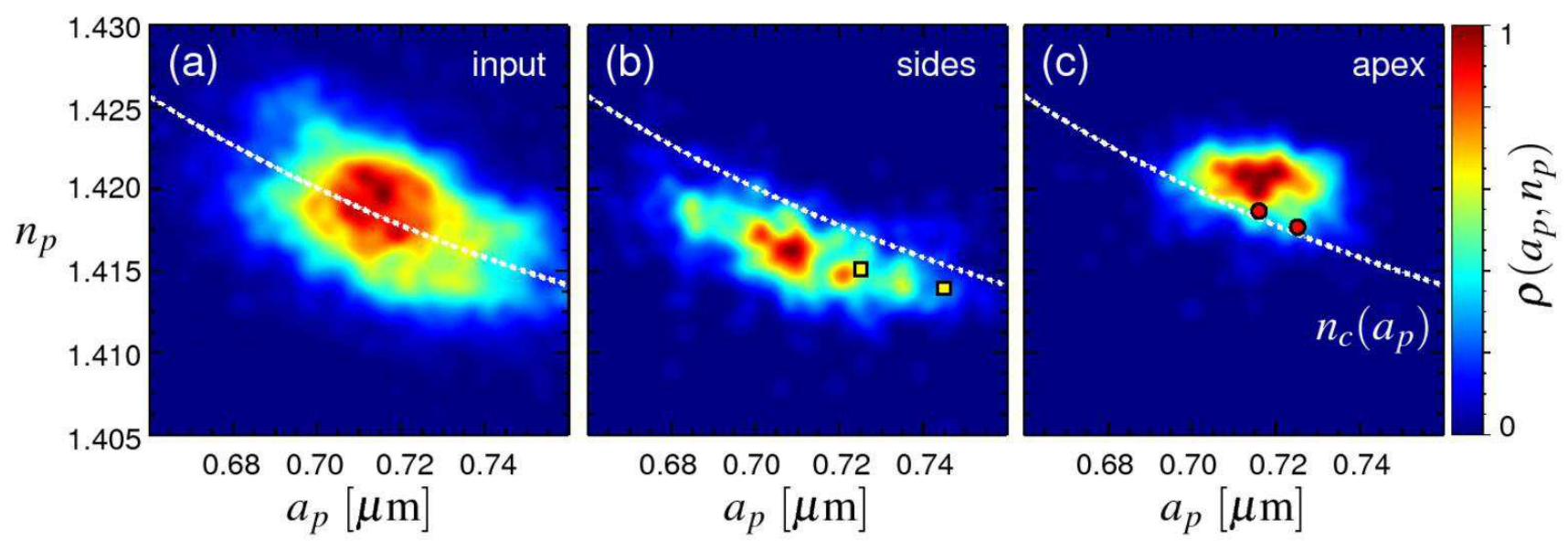

FIG. 2: (color online) High-resolution optical fractionation of monodisperse colloidal silica spheres demonstrated in the measured probability density $\rho\left(a_{p}, n_{p}\right)$ of particles with radius $a_{p}$ and refractive index $n_{p}$. The input sample, whose distribution is shown in (a), is separated into a smaller, low-index fraction in the side regions (b) and a larger, high-index fraction at the apex (c). The dashed curves show the marginally locked-in condition $n_{c}\left(a_{p}\right)$ for the array's [10] direction. Plotted squares and circles indicate the properties of the four representative spheres whose trajectories are plotted in Fig. 1(b).

depends on particle size through the ratio $a_{p} / b$ and

$$
B=\frac{\sqrt{e}}{2} \frac{6 \pi \eta v c}{n_{m} P} b \sin \theta
$$

depends only on control parameters. Particles satisfying $n_{p} \geq n_{c}\left(a_{p}\right)$ should become locked in to the lattice direction at angle $\theta$, and those with $n_{p}<n_{c}\left(a_{p}\right)$ should escape, perhaps to become locked in along other lattice directions at smaller angles. This result provides a basis to tune the selectivity of optical fractionation for sorting by size and refractive index.

The data in Fig. 2 show results obtained with a single sample of monodisperse silica spheres (PolySciences Catalog \#24327, Lot \#600424). Lorenz-Mie characterization of 6000 randomly chosen spheres yields a sampleaveraged radius of $a_{p}=0.715 \pm 0.021 \mu \mathrm{m}$, which is significantly smaller and more monodisperse than the manufacturer's specification of $0.75 \pm 0.04 \mu \mathrm{m}$. The sampleaveraged refractive index of $n_{p}=1.418 \pm 0.004$ is consistent with typical values for colloidal silica spheres [14]. The distribution of input particle properties in Fig. 2(a) was computed from single-particle results using an optimal non-parametric density estimator [25]. It reveals an anticorrelation between particle size and refractive index that is typical for porous colloidal spheres grown by emulsion polymerization and is not observed in fully compact particles such as fluid droplets [14].

The homogeneous input sample was flowed at $v=$ $34 \pm 2 \mu \mathrm{m} / \mathrm{s}$ through the array of traps. This is slow enough that blurring during the camera's $1 \mathrm{~ms}$ exposure time does not degrade holographic particle tracking and characterization [14]. Although particles approach the array at a range of heights and thus at a variety of speeds, they are drawn to the channel's midplane by optical forces, and thus pass through at a uniform speed.
This vertical flow focusing is made apparent by the threedimensional tracking capabilities of holographic video microscopy. The concentration of particles is low enough that no more than eight occupy the array at any time. Collisions are rare at this low occupancy and no effort was made to exclude their effects from our results.

The trap array's [10] lattice spacing of $b=2.03 \mu \mathrm{m}$ was chosen so that roughly half of the sample would be kinetically locked-in according to Eq. (4) with a laser power of $P=3 \mathrm{~mW} /$ trap. The critical condition for locked-in transport along the [10] lattice direction predicted by Eq. (4) is plotted as a dashed curve in Fig. 2 with no adjustable parameters. The [01] lattice spacing of $a=3.27 \mu \mathrm{m}$ was selected to be large enough to prevent locked-in particles from escaping [7], but small enough that the low-angle [11] direction would be able to entrain and deflect all of the particles in the sample. The array thus preferentially deflects particles toward its apex, but provides an alternative route with opposite deflection for particles that interact too weakly to lock in to its principal axis. The two fractions thus are spatially separated from each other and also from those particles that bypass the array's catchment basin altogether.

The characteristics of particles flowing into and out of the array were assessed within the rectangular regions indicated in Fig. 1(b), with results from the two side regions being combined. Particle-by-particle characterization in the side and apex regions, plotted in Figs. 2(b) and (c), reveals clear separation into two distinct and sharply defined sub-populations, the larger, higher-index particles being deflected to the apex and the smaller, lower-index particles being deflected to the sides, in excellent quantitative agreement with Eq. (44). The mean radius of the focused population is slightly larger than that of the 
other fraction $(0.716 \pm 0.021 \mu \mathrm{m}$ versus $0.710 \pm 0.027 \mu \mathrm{m})$. A far more substantial distinction is observed in the refractive indexes of the sorted populations. The focused spheres have a mean refractive index of $1.420 \pm 0.003$ whereas the dispersed fraction has a mean refractive index of $1.416 \pm 0.003$. The properties of the two spatially separated populations differ with better than 99.99 percent confidence according to the Wilcoxon rank-sum test.

The results in Fig. 2 demonstrate that optical fractionation is inherently a multidimensional sorting technique whose selectivity for size and refractive index can be dynamically optimized by tuning the properties of the optical trapping pattern. The present realization demonstrates discrimination on the basis of refractive index with part-per-thousand resolution under the set of conditions that we considered. Similarly good results, including agreement with Eq. (4), were obtained for a monodisperse sample of micrometer-diameter polystyrene spheres. These observations provides experimental support for the suggestion [7, 11, 26] that the large number of particle-trap interactions in a typical trajectory renders kinetic lock-in robust against the noise and quenched disorder that arise in practical implementations. A rigorous explanation for this robustness remains an outstanding challenge, as does the quantitative predictive power of Eq. (4), which was derived with semiquantitative limiting arguments [3, 7].

The inhomogeneous trapping pattern used for this study implements a prismatic mode of optical fractionation [1, 11] in which different fractions become kinetically locked in to different crystallographic directions and thus are sorted into spatially separated streams. More complex trapping patterns should enable still more sophisticated multi-dimensional sorting with similarly large displacements for finely resolved populations. The very high-resolution fractionation by refractive index that we have demonstrated should have immediate applications in photonics, where colloidal particles' optical properties increasingly are being exploited, and also in medical diagnostics, where pre-selecting probe particles for precise optical properties may reduce the time, effort and expense of bead-based molecular binding assays [14]. The throughput for such applications can be increased by proportionately increasing the flow speed and laser power.

This work was supported by the MRSEC program of the National Science Foundation through Grant Number DMR-0820341.

[1] P. T. Korda, M. B. Taylor, and D. G. Grier, Phys. Rev. Lett. 89, 128301 (2002).
[2] M. P. MacDonald, G. C. Spalding, and K. Dholakia, Nature 426, 421 (2003).

[3] K. Ladavac, K. Kasza, and D. G. Grier, Phys. Rev. E 70, 010901(R) (2004).

[4] A. Hirai, H. Monjushiro, and H. Watarai, Langmuir 12, 5570 (1996).

[5] A. Ashkin, J. M. Dziedzic, J. E. Bjorkholm, and S. Chu, Opt. Lett. 11, 288 (1986).

[6] Y. Roichman, B. Sun, Y. Roichman, J. Amato-Grill, and D. G. Grier, Phys. Rev. Lett. 100, 013602 (2008).

[7] M. Pelton, K. Ladavac, and D. G. Grier, Phys. Rev. E 70, 031108 (2004).

[8] J. M. Sancho, M. Khoury, K. Lindenberg, and A. M. Lacasta, J. Phys.: Condens. Matter 17, S4151 (2005).

[9] J. P. Gleeson, J. M. Sancho, A. M. Lacasta, and K. Lindenberg, Phys. Rev. E 73, 041102 (2006).

[10] A. M. Lacasta, M. Khoury, J. M. Sancho, and K. Lindenberg, Mod. Phys. Lett. B 20, 1427 (2006).

[11] A. Gopinathan and D. G. Grier, Phys. Rev. Lett. 92, 130602 (2004).

[12] G. Milne, D. Rhodes, M. MacDonald, and K. Dholakia, Opt. Lett. 32, 1144 (2007).

[13] Y. Roichman, V. Wong, and D. G. Grier, Phys. Rev. E 75, 011407 (2007).

[14] S.-H. Lee, Y. Roichman, G.-R. Yi, S.-H. Kim, S.-M. Yang, A. van Blaaderen, P. van Oostrum, and D. G. Grier, Opt. Express 15, 18275 (2007); F. C. Cheong, K. Xiao, and D. G. Grier, J. Dairy Sci. 92, 95 (2009); F. C. Cheong, B. Sun, R. Dreyfus, Amato-Grill, K. Xiao, L. Dixon, and D. G. Grier, Opt. Express 17, 13071 (2009).

[15] J. Sheng, E. Malkiel, and J. Katz, Appl. Opt. 45, 3893 (2006); S.-H. Lee and D. G. Grier, Opt. Express 15, 1505 (2007).

[16] D. C. Duffy, J. C. McDonald, O. J. A. Schueller, and G. M. Whitesides, Acta Crystallographica 70, 4974 (1998).

[17] E. R. Dufresne and D. G. Grier, Rev. Sci. Instrum. 69, 1974 (1998); D. G. Grier, Nature 424, 810 (2003).

[18] M. Polin, K. Ladavac, S.-H. Lee, Y. Roichman, and D. G. Grier, Opt. Express 13, 5831 (2005).

[19] Y. Roichman, I. Cholis, and D. G. Grier, Opt. Express 14, 10907 (2006).

[20] Y. Roichman, A. S. Waldron, E. Gardel, and D. G. Grier, Appl. Opt. 45, 3425 (2006).

[21] C. F. Bohren and D. R. Huffman, Absorption and Scattering of Light by Small Particles (Wiley Interscience, New York, 1983).

[22] J. Herrmann, M. Karweit, and G. Drazer, Phys. Rev. E 79, 061404 (2009).

[23] Y. Harada and T. Asakura, Opt. Commun. 124, 529 (1996).

[24] J. Happel and H. Brenner, Low Reynolds Number Hydrodynamics (Kluwer, Dordrecht, 1991).

[25] B. W. Silverman, Density Estimation for Statistics and Data Analysis (Chapman \& Hall, New York, 1992).

[26] P. T. Korda, Ph.D. thesis, The University of Chicago (2002). 